\title{
Repercussões emocionais e processos adaptativos vividos por pessoas estomizadas
}

\author{
Emotional repercussions and adaptive processes lived by stomized people \\ Repercusiones emocionales y procesos adaptativos vividos por personas estomizadas
}

\begin{abstract}
RESUMO
Objetivo: Mapear a produção científica sobre as repercussões emocionais e os processos adaptativos vividos por pessoas com estomias. Método: Revisão integrativa com coleta de dados nos meses de julho a agosto, nas bases de dados dos sítios da Biblioteca Virtual em Saúde, Literatura Latino Americana e do Caribe em Ciências da Saúde, na Literatura Internacional em ciência da Saúde e na Base de Dados de Enfermagem, com os escritores "emoções", "sentimentos", "estomias", "pessoas" e "cuidados de Enfermagem". Utilizaram-se artigos publicados entre os anos 2013 a 2017. Resultados: Com análise temática emergiram três categorias: "repercussões emocionais e as dificuldades no processo de adaptação da pessoa com estomia", "medidas de enfrentamento no processo de reabilitação da pessoa com estomia" e "o cuidado de enfermagem: apoio profissional relacionado ao autocuidado". Conclusão: A pessoa com estomia vivenciou momentos de depressão, tristeza, isolamento social e afastamento da atividade laboral. 0 enfermeiro em conjunto com a equipe multidisciplinar precisa desenvolver além de planos de cuidados e orientações, acompanhamento psicológico e apoio emocional para que a pessoa com estomia tenha uma melhor adaptação.
\end{abstract}

DESCRITORES: Emoções; Sentimentos; Estomias; Pessoas; Cuidados de Enfermagem.

\section{ABSTRACT}

Objective: To map the scientific production on the emotional repercussions and adaptive processes experienced by people with stomas. Method: Integrative review with data collection from July to August, in the databases of the Virtual Health Library, Latin American and Caribbean Literature in Health Sciences websites, in the International Literature in Health Science and in the Database of Nursing, with the writers "emotions", "feelings", "stomas", "people" and "Nursing care". Articles published between the years 2013 to 2017 were used. Results: With thematic analysis, three categories emerged: "emotional repercussions and difficulties in the adaptation process of the person with a stoma", "coping measures in the process of rehabilitation of the person with a stoma" and "nursing care: professional support related to self-care". Conclusion: The person with an ostomy experienced moment of depression, sadness, social isolation, and withdrawal from work activity. The nurse in conjunction with the multidisciplinary team needs to develop, in addition to care plans and guidelines, psychological support and emotional support so that the person with a stoma has a better adaptation.

DESCRIPTORS: Emotions; Feelings; Ostomies; People; Nursing Care.

\section{RESUMEN}

Objetivo: Mapear la producción científica sobre las repercusiones emocionales y los procesos adaptativos que experimentan las personas con estomas. Método: Revisión integrativa con recolección de datos de julio a agosto, en las bases de datos de la Biblioteca Virtual en Salud, sitios web de Literatura Latinoamericana y del Caribe en Ciencias de la Salud, en la Literatura Internacional en Ciencias de la Salud y en la Base de Datos. de Enfermería, con los escritores "emociones", "sentimientos", "estomas", "personas" y "Atención de enfermería". Se utilizaron artículos publicados entre los años 2013 a 2017. Resultados: Con el análisis temático surgieron tres categorías: "repercusiones emocionales y dificultades en el proceso de adaptación de la persona con estoma", "medidas de afrontamiento en el proceso de rehabilitación de la persona con estoma". y "cuidados de enfermería: apoyo profesional relacionado con el autocuidado". Conclusión: La persona con ostomía experimentó momentos de depresión, tristeza, aislamiento social y retraimiento de la actividad laboral. El enfermero junto con el equipo multidisciplinar necesita desarrollar, además de planes y pautas de atención, apoyo psicológico y apoyo emocional para que la persona con estoma tenga una mejor adaptación.

DESCRIPTORES: Emociones Sentimientos; Ostomías; Personas; Cuidado de Enfermería.

RECEBIDO EM: 14/02/2020 APROVADO EM: 15/02/2020 


\section{Hosana Pereira Cirino}

Enfermeira Estomaterapeuta UERJ. Mestre em Enfermagem pelo programa de Pós-graduação em Enfermagem da Universidade do Estado do Rio de Janeiro- UERJ.

ORCID:

\section{Priscila Cristina da Silva Thiengo de Andrade}

Enfermeira. Professora Assistente do departamento Médico-Cirúrgico da Faculdade de Enfermagem UERJ e Doutoranda em Enfermagem pelo programa de Pós-graduação em Enfermagem da Universidade do Estado do Rio de Janeiro- UERJ.

ORCID:

\section{Celia Caldeira Fonseca Kestenberg}

Enfermeira e Psicóloga Clínica, Professora Associada da UERJ em Enfermagem Psiquiátrica e Saúde Mental, Doutora em Psicologia Social.

ORCID:

\section{Célia Pereira Caldas}

Professora Titular do Departamento de Enfermagem de Saúde Pública da Faculdade de Enfermagem UERJ, Doutora em Enfermagem.

ORCID:

\section{Cleia do Nascimento Santos}

Enfermeira em Gerontologia UERJ. Mestre em Enfermagem pelo programa de Pós-graduação em Enfermagem da Universidade do Estado do Rio de Janeiro- UERJ.

ORCID:

\section{Wanderson Alves Ribeiro}

Enfermeiro Estomaterapeuta UERJ. Professor do Curso de Graduação em Enfermagem da UNIG/UCB. Mestre em Ciências do Cuidado em Saúde pela Universidade Federal Fluminense-UFF.

ORCID:

\section{INTRODUÇÃO}

A estomização é um procedimento cirúrgico agressivo que pode provocar várias mudanças na fisiologia corporal, no estilo de vida, no aspecto físico e psicossocial da pessoa. Podendo causar modificações na vida, na autoestima e na autoimagem ${ }^{(1)}$.

Estomia é a abertura ou criação de uma boca, em órgão oco, através de procedimento cirúrgico, a fim de desviar o trânsito gástrico ou intestinal, para alimentação ou eliminaçãa ${ }^{(2)}$.

Dentre as diversas causas que podem levar à necessidade da confecção de uma estomia intestinal, destacam-se as doenças diverticulares e inflamatórias intestinais, como: Doença de Crohn e colite ulcerativa, além de câncer colorretal, traumas abdominais e anomalias colorretais ${ }^{(3)}$.

Cabe ressaltar que, após a realização da estomia, a pessoa vive sentimentos de medo, raiva e depressão devido à alteração da sua imagem corporal ou luto, necessitando de apoio para facilitar a sua aceitação e adaptação à nova realidade, já que que a estomização interfere diretamente na autoimagem e na autoestima ${ }^{(4)}$.

Nesse sentido, estudo ${ }^{(1)}$ ainda ressalta que a beleza e o vigor são supervalorizados em nossa sociedade, sendo a cirurgia de estomização considerada um desvio dos padrões de normalidade, podendo gerar significativo senso de rejeição, onde a pessoa vivencia a desconstrução de sua imagem, de sua posição e de sua função social, podendo apresentar sentimentos conflituosos que, por sua vez, podem inibir o processo de adaptação e aceitação da sua nova condição de vida.

Nesse sentido, investigar as questões emocionais nos fará ver os estomizados como um todo e, ao mesmo tempo, como um ser individual, facilitando o processo de adaptação e aceitação de sua nova condição.

Vive-se, atualmente, em uma sociedade que cultua o corpo e a boa aparência, onde a beleza ganha espaço para a aceitação ou rejeição, na qual não ser belo equivale a ser rejeitado. É imposto pela sociedade um corpo padrão para o cotidiano. Para viver, nesse sentido, é necessário expor a aparência física, assumindo funções e poderes que dão acesso ao mundo, exigindo a presença corporal um do outro ${ }^{(5)}$.

Nesse cenário, o objetivo deste estudo foi mapear a produção científica sobre as repercussões emocionais e sentimentos vividos por pessoas estomizadas. $\mathrm{O}$ conhecimento de emoções e sentimentos gerados pela estomização auxilia na elaboração de ações voltadas especificamente para as necessidades dessa clientela, reforçando a atuação da equipe de enfermagem na orientação ao autocuidado, reinserção desse paciente ao convívio social, retorno à vida laboral, melhora da autoestima e autoimagem.

Ressalta-se que este tema é de extrema relevância devido à necessidade de conhecer as repercussões emocionais e sentimentos sofridos por pacientes es- 
tomizados, com o intuito de melhorar a assistência a essa clientela, facilitando sua adaptação à nova realidade e contribuindo para o autocuidado. Difundir informações para um atendimento mais completo e humanizado desta clientela é muito importante, visto que os esforços são, em sua maioria, direcionados à correta utilização da bolsa coletora, da manutenção da pele e do estoma.

\section{METODOLOGIA}

Para fundamentar este estudo e compreender a lacuna de conhecimento, foi realizada uma revisão integrativa da literatura. Esta modalidade de estudo foi escolhida, pois corresponde à uma técnica em que são reunidas e organizadas pesquisas desenvolvidas e concluídas por especialista, sendo analisados os achados de estudos, primando-se pelo rigor e sistemática, examinando os métodos e estratégias empregadas, apreciando as fontes e sintetizando os resultados ${ }^{(6)}$.

A revisão integrativa da literatura permite ao pesquisador se aproximar da problemática que deseja investigar, definindo um panorama sobre a sua produção científica, de modo que se conheça a evolução do tema ao longo do tempo e, com isso, conceber possíveis oportunidades de pesquisa ${ }^{(7)}$.

\section{0 estabelecimento do problema de revisão}

Deve-se iniciar com a definição de um problema e a formulação de uma pergunta de pesquisa. Para isso, foi utilizada como ferramenta a estratégia PICO, definida como uma forma de maximizar a recuperação de evidências nas bases de dados, mantendo o foco na finalidade da pesquisa e evitando a realização de buscas desnecessárias ${ }^{(8)}$.

Diante do exposto, o presente estudo foi direcionado pelo seguinte questionamento:

Quadro 1. Descrição da estratégia PICO. Rio de Janeiro, RJ, Brasil, 20113-2017

\begin{tabular}{|l|l|}
\hline P (Paciente ou Problema) & Pessoas portadoras de estomias de eliminação. \\
\hline I (Intervenção) & A confecção cirúrgica de uma estomia. \\
\hline C (Controle ou Comparação) & Não se aplica, por não se tratar de um estudo comparativo. \\
\hline O (Desfechos ou "Outcomes") & Sentimentos vividos por pessoas estomizadas. \\
\hline
\end{tabular}

processos adaptativos vividos por pessoas estomizadas?".

\section{A seleção da amostra}

Optou-se pelos seguintes descritores

de assunto nos

campos de busca

das bases de dados:

"sentimentos,

adaptação, estomia,

pessoas e cuidados

de enfermagem",

correlacionando-os

no sentido de refinar

a população do

estudo. Essa busca

ocorreu no mês de

junho de 2018.

"Quais as evidências científicas dis-
poníveis na literatura, publicadas em
periódicos on-line, da área de enferma-
gem, relacionadas aos sentimentos e aos

dast

\section{interter} envolvendo revisões de literatura. Foram excluídos teses, dissertações, cartas e artigos repetidos. 


\section{artigo}

Citino, H.P.; Andrade, P.C.S.T.; Kestenberg, C.C.F.; Caldas, C.P.; Santos, C.N.; Ribeiro, W.A.

Repercussões emocionais e processos adaptativos vividos por pessoas estomizadas

Para a consulta nas bases de dados da BVS, fez-se uso dos seguintes agrupamentos de descritores: "Estomia/ostomia and Pessoas", "Estomia/Ostomia and Sentimentos",
"Estomia/Ostomia and Pessoas", "Estomia/ Ostomia and Cuidados de Enfermagem".

Diante do exposto, cabe mencionar que partes dos artigos selecionados se re-

\section{Fluxograma 1. Referente à busca. Rio de Janeiro, RJ, Brasil, 2013-2017}

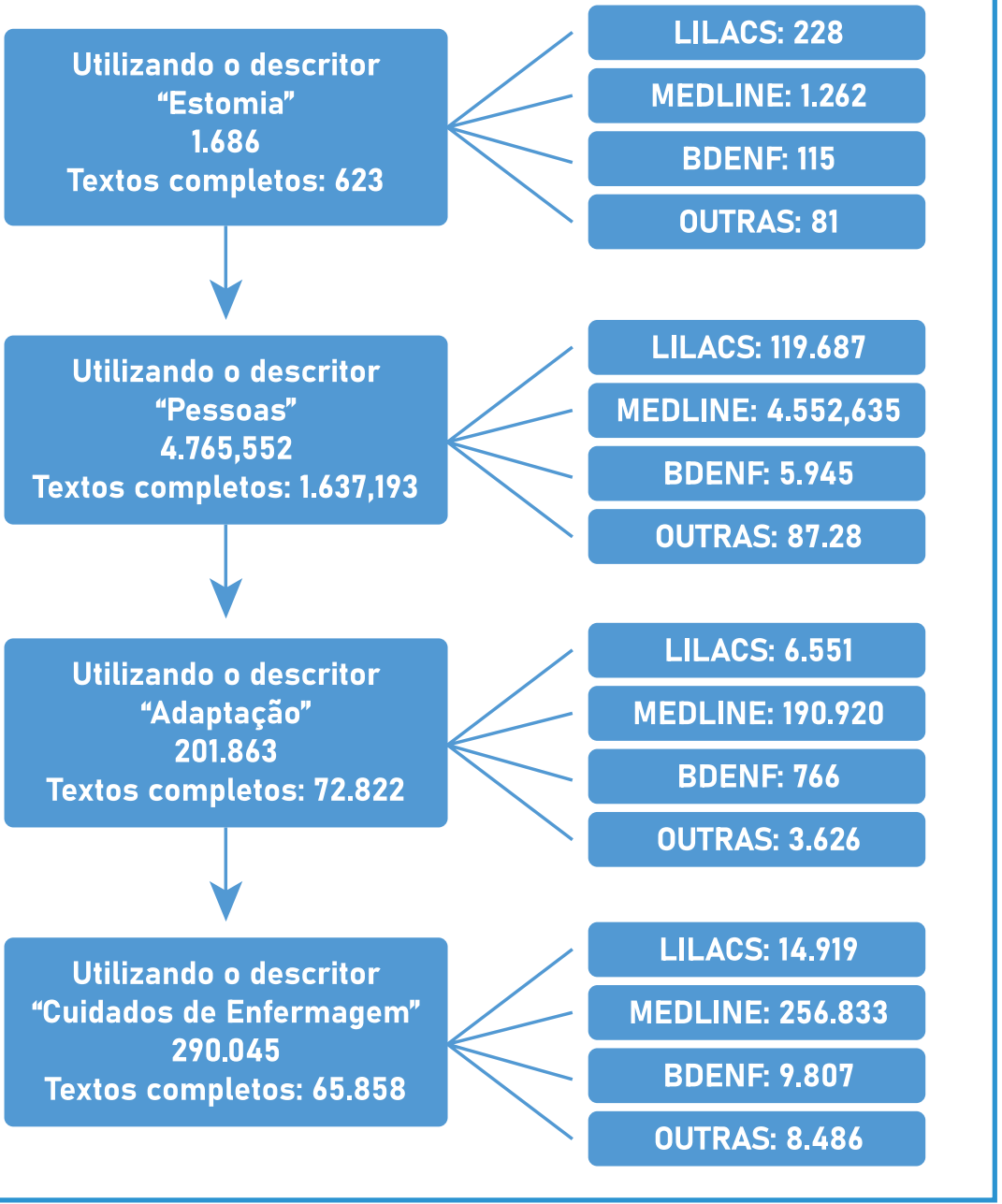

petiam entre as buscas com os descritores nas combinações supracitadas e nas bases de dados expostas acima.

Após a leitura reflexiva dos achados, procedeu-se com a categorização dos estudos.

\section{A categorização dos estudos}

Essa é a terceira fase, em que os dados são coletados após a seleção dos artigos. Nessa fase, é necessária a utilização de um instrumento de coleta de dados previamente elaborado que seja capaz de extrair os dados relevantes, reduzindo os riscos de $\operatorname{erros}^{(10)}$.

Um estudo pode ser caracterizado pela utilidade direta dos achados. A pesquisa básica é aplicada para acumular informações, expandindo a base de conhecimento para melhorar a compreensão, ou para a obtenção de informações pertinentes ao estudo ${ }^{(11)}$.

Para organizar a obtenção das informações contidas nos estudos analisados, foi utilizado um instrumento de coleta de dados produzido pela mestranda, o qual permitiu o recolhimento de informações de modo sistemático. Este instrumento foi formatado em quatro divisórias a fim de otimizar a síntese dos artigos e facilitar a leitura.

A primeira divisória do instrumento de coleta de dados é composta por dados referentes à publicação, como o autor, o título do artigo, o nome do periódico e o ano de publicação. A segunda divisória contém informações referentes ao tipo de estudo ou técnica utilizada (dados da metodologia empregada). A terceira se refere aos achados da pesquisa (o que o estudo apresentou de resultados). Já a quarta par-

Quadro 2. Descritores associados para busca e refinamento da pesquisa. Rio de Janeiro, RJ, Brasil, 2013-2017

\begin{tabular}{|l|c|c|c|c|}
\hline PALAVRAS-CHAVE & ENCONTRADOS & $\begin{array}{c}\text { APLICAÇÃO DE CRITÉRIOS DE } \\
\text { INCLUSÃO }\end{array}$ & $\begin{array}{c}\text { APLICAÇÃO DE CRITÉRIOS } \\
\text { DE EXCLUSÃO }\end{array}$ & $\begin{array}{c}\text { ARTIGOS } \\
\text { SELECIONADOS }\end{array}$ \\
\hline $\begin{array}{l}\text { Estomia/ostomia and } \\
\text { Pessoas }\end{array}$ & 516 & 41 & 475 & 15 \\
\hline $\begin{array}{l}\text { Estomia/Ostomia and } \\
\text { Sentimentos }\end{array}$ & 28 & 16 & 12 & 03 \\
\hline $\begin{array}{l}\text { Estomia/Ostomia and } \\
\text { Adaptação }\end{array}$ & 124 & 95 & 27 & 02 \\
\hline $\begin{array}{l}\text { Estomia/Ostomia and Cui- } \\
\text { dados de Enfermagem }\end{array}$ & 872 & 17 & 855 & 03 \\
\hline Total & & & & 23 \\
\hline
\end{tabular}


te apresenta a base de dados em que o estudo foi localizado.

Após a organização dos achados e a leitura, os estudos foram categorizados de acordo com as temáticas apresentadas.

Após a análise dos dados, emergiram três temáticas ou categorias, denominadas por: Repercussões emocionais e as dificuldades no processo de adaptação da pessoa com estomia; Medidas de enfrentamento no processo de reabilitação da pessoa com estomia; e $\mathrm{O}$ cuidado de enfermagem: apoio profissional relacionado ao autocuidado.

\section{RESULTADOS}

O quadro a seguir apresenta os artigos selecionados para a pesquisa.

\section{Quadro 3. Síntese dos artigos resultados da revisão integrativa. Rio de Janeiro, RJ, Brasil, 2013-2017}

\begin{tabular}{|l}
\hline AUTOR, TÍTULO, PERIÓDICO E ANO \\
\hline $\begin{array}{l}\text { Aguiar et. al., Aspectos sociodemográfi- } \\
\text { cos e clínicos de estomizados intestinais } \\
\text { provisórios. REME, } 2017 .\end{array}$ \\
\hline
\end{tabular}

Freire et al., Autoimagem e autoconceito na vivência de pacientes estomizados: Olhar da Enfermagem. REME, 2017.

Barba et al., Demanda de cuidados de pacientes oncológicos estomizados na atenção primária à saúde. Rev Enf UFPE, 2017.

Costa et. al., Distúrbio na imagem corporal: Diagnóstico de enfermagem e características definidoras em pessoas ostomizadas. AQUICHAN, 2017.

Vera et. al., Sexualidade de pacientes com estomias intestinais de eliminação. Ver Pesq. Cuid. Fundam. 2017.

Silva et. al., Qualidade de vida de pessoas com estomias intestinais de eliminação. Acta Paul. Enferm. 2017.

Mohr et al., Adolescent perspective following Ostomy Surgery: A Grounded Theory Study 2016. J Wound Ostomy Continence Nurs. 2016.

Melo et. al., Revisão integrativa das características definidoras do diagnóstico de enfermagem: Disposição para resiliência melhorada em ostomizados. REME, 2015.

Simon et. al., "Sempre ajudando em uma coisa ou outra": Rede Social da família da pessoa com estomia. Ver. Eletr. Enferm. 2014.

Torres et. al., Qualidade de vida de pessoas estomizadas: Revisão Integrativa. Rev. Enferm. UFPI. 2015.
PROCEDIMENTO METODOLÓGICO

ACHADOS DA PESQUISA

Alteração da imagem que o indivíduo tem de si, senti-

Transversal

quantitativo

mentos negativos, inferioridade, alterando a sexualidade, autoimagem, autoestima e emoções.

O uso da colostomia está ligado a sentimentos de

vergonha, medo, insegurança, invasão e sofrimento, refletindo na vida social, amorosa e laboral.

Mudanças com a presença do estoma e sentimentos

Exploratório

Descritivo

qualitativo

como medo, incertezas e preocupações, necessidades dos

Descritivo

qualitativo profissionais de enfermagem em desenvolver ações de educação em saúde para o paciente adquirir autonomia.

O estudo revelou o diagnóstico de enfermagem "Distúrbio da imagem corporal", comportamento de monitorar o próprio corpo, impacto na qualidade de vida, na sexualidade,

integrativa

descritiva

qualitativa no estilo de vida e no envolvimento social.

A estomia influencia em diversos fatores que contribuem para prejudicar o bem-estar das pessoas. A mudança na

Exploratório descritivo quantitativo imagem corporal altera as funções psíquicas, influencian-

LILACS do a imagem sexual.

Descritivo transversal

Estomias intestinais de eliminação interferem na qualidade de vida, principalmente nos âmbitos físicos e social.

LILACS

A experiência da estomia inclui conceitos do "eu físico" e "eu social" com o objetivo de normalização. As análises incluem o eu físico, mudança de realidade, aprender, adaptar-se ao dia-a-dia e a vida social.

Verificou-se que o diagnóstico de Enfermagem "disposição para resiliência melhorada" pode ser identificado em pessoas estomizadas, visto que das 17 características definidoras desse diagnóstico, 10 foram encontradas na literatura para tal população.

A formação da rede social dessas famílias se organiza por várias teias composta pela própria família que possibilita a troca de apoio e fortalecimento das relações, ajudando a familia no cuidado. Essas redes contribuem para o reconhecimento da autoimagem, bem-estar, cuidado, enfrentamento e adaptação.

O estudo destaca a importância da implantação e permanência de uma equipe multidisciplinar e reflexiva, sendo embasada na visão holística, visando a reinserção social

BASE DE DADOS

BDENF

BDENF

BDENF

LILACS

Exploratório

descritivo

qualitativo
LILACS

\section{LILACS}

BDENF e o fornecimento de estratégias de enfrentamento e adaptação.

Revisão

Integrativa 


\section{artigo}

Citino, H.P.; Andrade, P.C.S.T.; Kestenberg, C.C.F.; Caldas, C.P.; Santos, C.N.; Ribeiro, W.A.

Repercussões emocionais e processos adaptativos vividos por pessoas estomizadas

\begin{tabular}{|c|c|c|c|}
\hline $\begin{array}{l}\text { Sena et. al., Perfil dos urostomizados } \\
\text { cadastrados em uma associação de osto- } \\
\text { mizados. Cogitare Enfermagem. } 2014 .\end{array}$ & $\begin{array}{l}\text { Descritivo } \\
\text { Retrospectivo } \\
\text { Consulta } \\
\text { documental }\end{array}$ & $\begin{array}{c}\text { O estudo do perfil de pacientes urostomizados auxiliou a } \\
\text { prática clínica, com a identificação das especificidades da } \\
\text { população de ostomizados, colaborando com a promoção } \\
\text { da saúde. }\end{array}$ & LILACS \\
\hline $\begin{array}{l}\text { Maurício et. al., Determinantes biopsicos- } \\
\text { sociais do processo de inclusão laboral da } \\
\text { pessoa estomizada. REBEN. } 2014 .\end{array}$ & $\begin{array}{l}\text { Exploratório } \\
\text { descritivo } \\
\text { qualitativo }\end{array}$ & $\begin{array}{l}\text { Conhecer mais profundamente as facilidades e dificulda- } \\
\text { des dos estomizados em relação à inclusão laboral. Os } \\
\text { fatores dificultadores perpassam no âmbito físico/biológi- } \\
\text { co e psicossocial. }\end{array}$ & MEDLINE \\
\hline $\begin{array}{l}\text { Simon et. al., Configuração da rede de } \\
\text { assistência às pessoas com estomia: } \\
\text { Interface do cuidado contínuo. J Nurs. } \\
\text { Healt. } 2014 \text {. }\end{array}$ & $\begin{array}{l}\text { Revisão } \\
\text { Integrativa }\end{array}$ & $\begin{array}{l}\text { Poucas publicações sobre a temática e as existentes } \\
\text { revelam uma assistência fragmentada, em que o sujeito } \\
\text { com estomia e sua família necessitam interconectar os } \\
\text { múltiplos pontos da rede de saúde para conseguir assis- } \\
\text { tência continuada. }\end{array}$ & BDENF \\
\hline $\begin{array}{l}\text { Barros et. al., Ações ecossistêmicas e } \\
\text { gerontotecnológicas no cuidado de enfer- } \\
\text { magem complexo ao idoso estomizado. } \\
\text { Ver. Bras. Enf. } 2014 \text {. }\end{array}$ & $\begin{array}{l}\text { Descritivo } \\
\text { qualitativo }\end{array}$ & $\begin{array}{l}\text { Identificou-se ações ecossistêmicas e gerontotecnológi- } \\
\text { cas como possibilidades de um novo olhar a cerca de si e } \\
\text { do outro, em que o idoso estomizado percebe as contra- } \\
\text { dições entre capacidade/incapacidade, ordem/desordem, } \\
\text { motivação/frustração e assim estabelece medidas de } \\
\text { enfrentamento. }\end{array}$ & MEDLINE \\
\hline $\begin{array}{l}\text { Ardigo et. al., Conhecimento do profissio- } \\
\text { nal acerca do cuidado de enfermagem à } \\
\text { pessoa com estomia intestinal e familia. } \\
\text { Texto \& Contexto Enf. } 2013 .\end{array}$ & $\begin{array}{l}\text { Exploratório } \\
\text { descritivo } \\
\text { qualitativo }\end{array}$ & $\begin{array}{l}\text { Os resultados mostram que as pessoas com estomia, } \\
\text { assim como a familia apresentam sentimentos de rejei- } \\
\text { ção e medo. Esses sentimentos negativos dificultam o } \\
\text { aprendizado do autocuidado e da adaptação à sua nova } \\
\text { condição. Pacientes orientados no pré-operatório adap- } \\
\text { tam-se melhor. }\end{array}$ & LILACS \\
\hline $\begin{array}{l}\text { Vilar et. al., O cuidar da criança estomiza- } \\
\text { da no domicílio: estudo de caso: Online B. } \\
\text { J. Nurs. } 2013 .\end{array}$ & $\begin{array}{l}\text { Descritivo } \\
\text { qualitativo }\end{array}$ & $\begin{array}{l}\text { A participação não somente do enfermeiro, mas da equipe } \\
\text { multidisciplinar é fundamental na orientação para a alta } \\
\text { hospitalar ao cuidado da criança estomizada. }\end{array}$ & BDENF \\
\hline $\begin{array}{l}\text { Coelho et. al., A estomia mudando a vida: } \\
\text { Enfrentar para viver. REME } 2013 .\end{array}$ & $\begin{array}{l}\text { Exploratório } \\
\text { descritivo } \\
\text { qualitativo }\end{array}$ & $\begin{array}{l}\text { Os estomizados apresentam mudanças no seu modo de } \\
\text { vida após a confecção do estoma, sendo essas mudanças } \\
\text { provenientes de alterações físicas e sociais. }\end{array}$ & LILACS \\
\hline $\begin{array}{l}\text { Souza et. al., O preparo do familiar para o } \\
\text { cuidado à pessoa com estomia. Ver. Enf. } \\
\text { UFPE. } 2013 .\end{array}$ & $\begin{array}{l}\text { Descritivo } \\
\text { qualitativo }\end{array}$ & $\begin{array}{l}\text { O familiar cuidador da pessoa com estomia é despre- } \\
\text { parada para o cuidado e sem conhecimento acerca do } \\
\text { processo de estomização, mas assume o cuidado, apesar } \\
\text { de seu próprio despreparo. }\end{array}$ & BDENF \\
\hline $\begin{array}{l}\text { Teles et. al., Mudanças físicas, psicos- } \\
\text { sociais e os sentimentos gerados pela } \\
\text { estomia intestinal para o paciente. Ver. } \\
\text { Enf. UFPE. } 2017\end{array}$ & $\begin{array}{l}\text { Revisão } \\
\text { integrativa }\end{array}$ & $\begin{array}{l}\text { Evidenciaram-se artigos focalizando os sentimentos } \\
\text { relatados pelos pacientes, sendo esses de surpresa, an- } \\
\text { siedade, incerteza, medo do diagnóstico e da morte, raiva, } \\
\text { desespero, angústia, rejeição e impotência. }\end{array}$ & BDENF \\
\hline $\begin{array}{l}\text { Goulart et. al., A sexualidade do paciente } \\
\text { estomizado no discurso do Enfermeiro. } \\
\text { REME. } 2017 .\end{array}$ & $\begin{array}{l}\text { Qualitativa Análise } \\
\text { de conteúdo }\end{array}$ & $\begin{array}{l}\text { Destacados aspectos como a compreensão da sexua- } \\
\text { lidade na visão da equipe de enfermagem, a formação } \\
\text { acadêmica, os sentimentos ao discutir os temas, a assis- } \\
\text { tência ao estomizado não atinge a sua plenitude, apenas } \\
\text { direcionada ao estoma. }\end{array}$ & BDENF \\
\hline $\begin{array}{l}\text { Wild et. al., Educação em saúde com } \\
\text { estomizados e seus familiares: Possibili- } \\
\text { dade para melhor qualidade de vida. Rev. } \\
\text { Enf. UFSM. } 2016\end{array}$ & $\begin{array}{l}\text { Relato de } \\
\text { experiência }\end{array}$ & $\begin{array}{l}\text { No grupo, foram abordados assuntos relativos à alimenta- } \\
\text { ção, sexualidade, autocuidado e autoimagem, a proposta } \\
\text { contribuiu para a melhoria da qualidade de vida desses } \\
\text { pacientes. }\end{array}$ & BDENF \\
\hline $\begin{array}{l}\text { Mauricio et. al., O enfermeiro e sua par- } \\
\text { ticipação no processo de reabilitação da } \\
\text { pessoa com estomia. Esc. Anna Nery Ver. } \\
\text { Enf. } 2013\end{array}$ & $\begin{array}{l}\text { Exploratório } \\
\text { descritivo } \\
\text { qualitativo }\end{array}$ & $\begin{array}{l}\text { Poucos estomizados receberam orientações pelos profis- } \\
\text { sionais de enfermagem a respeito do retorno às ativida- } \\
\text { des trabalhistas, há lacunas no processo. }\end{array}$ & LILACS \\
\hline
\end{tabular}




\begin{tabular}{|l|c|c|c|}
\hline $\begin{array}{l}\text { Menezes et. al., A subjetividade no cuida- } \\
\text { do familiar à criança ostomizada a partir } \\
\text { da construção de sua autonomia. }\end{array}$ & $\begin{array}{c}\text { Exploratório } \\
\text { descritivo } \\
\text { qualitativo }\end{array}$ & $\begin{array}{c}\text { A vivência da família diante de uma nova realidade impos- } \\
\text { ta pelo tratamento desencadeia reflexos de sensações } \\
\text { consideradas subjetividade, envolvendo nuances culturais } \\
\text { inerentes a cada ser, tornando importante a aliança de } \\
\text { saberes entre profissional e cliente. }\end{array}$ & LILACS \\
\hline $\begin{array}{l}\text { Nichols et. al., Quality of life in Us resi- } \\
\text { dents with ostomies assed. }\end{array}$ & $\begin{array}{c}\text { Análise em banco } \\
\text { de dados }\end{array}$ & $\begin{array}{c}\text { Pacientes estomizados demonstraram niveis de dificulda- } \\
\text { des, adoecimentos e limitações em atividades físicas. }\end{array}$ & LILACS \\
\hline $\begin{array}{l}\text { Seardillo et. al., Eploring the relationship } \\
\text { between resilience and ostomy ad- } \\
\text { justment in adults with a permanente } \\
\text { ostomy. J Wound Ostomy Continence } \\
\text { Nurs. } 2016\end{array}$ & $\begin{array}{c}\text { Descritivo } \\
\text { Pesquisa de } \\
\text { campo }\end{array}$ & $\begin{array}{c}\text { Os participantes com níveis altos de ajustes da estomia } \\
\text { apresentaram niveis mais elevados de resiliência e os } \\
\text { mais resilientes, relataram níveis mais altos de adaptação. }\end{array}$ & MEDLINE \\
\hline $\begin{array}{l}\text { Queiroz et. al., Autoestima em mujeres } \\
\text { com ostomias intestinales. Index Enfer- } \\
\text { meria. 2017 }\end{array}$ & $\begin{array}{c}\text { Estudo } \\
\text { Quantitativo } \\
\text { Transversal } \\
\text { analítico }\end{array}$ & $\begin{array}{c}\text { A autoestima das mulheres estomizadas pode melhorar } \\
\text { de acordo com suas características sociodemográficas, clí- } \\
\text { nicas e de autocuidado, portanto esses fatores devem ser } \\
\text { considerados para desenvolvimento de ações multiprofis- } \\
\text { sionais no seu enfrentamento. }\end{array}$ & MEDLINE \\
\hline
\end{tabular}

\section{DISCUSSÃO}

Para realizar a análise dos dados das pesquisas, faz-se necessária uma abordagem organizada para ponderar o rigor e as características de cada estudo. Nesta fase, a experiência do pesquisador contribui na apuração da validade dos métodos e da análise dos resultados ${ }^{(10)}$.

Os critérios para a revisão integrativa envolvem parâmetros minuciosos de análise, utilizando métodos que garantam o alcance dos objetivos: aplicar análise rigorosa, examinar a teoria, estabelecer relações com os resultados, métodos, sujeitos e atributos da pesquisa, com a intenção de apresentar ao leitor informações sobre os estudos revisados, sem destacar apenas os resultados, melhorando as possíveis informações.

Após a leitura reflexiva dos achados, procedeu-se com a categorização dos estudos.

\section{Repercussões emocionais e as difi- culdades no processo de adaptação da pessoa com estomia}

Nesta categoria, evidenciaram-se artigos que destacam as mudanças no estilo de vida, as emoções e os sentimentos vivenciados pelos pacientes estomizados, provocados após a confecção da estomia. As repercussões acarretadas por essas alteraçôes podem fazer emergir sentimentos que influenciarão em sua adaptação, principalmente quando relacionados a mudanças que interferem diretamente nas atividades de vida diária, como relacionamentos, trabalho, lazer, entre outras.

Após a reação inicial da confecção do estoma, visualizam-se no sujeito momentos de depressão e tristeza, geralmente ocasionados pela perda do prazer em realizar atividades normalmente valorizadas. As modificações no estilo de vida da pessoa estomizada por conta da alteração da anatomia e da função fisiológica reduzem a capacidade desses pacientes, impondo- -lhes limites ${ }^{(12)}$.

Segundo estudo ${ }^{(4)}$, estomizados apresentam, também, sentimentos como angústia, medo, dificuldade de aceitação da situação e dificuldade de adaptação à essa nova condição de saúde. Podem sentir, ainda, rejeição como defesa a essa não aceitação, que podem vir a sofrer das pessoas que os cercam, com sentimentos de incapacidade e desprestígio.

As questões psicossociais foram abordadas por autores ${ }^{(13)}$, que afirmam que a estomização afeta os sentimentos do paciente, implicando na qualidade de vida. A presença do estoma e da bolsa coletora conflita com a imagem corporal ideal imposta pela sociedade.

Nesse contexto, estudo ${ }^{(14)}$ discorre que estabelecer um cuidado com a bolsa no dia a dia é algo que faz parte da doutrina do ser, pois tem um significado muito importante para os pacientes. As preocupações advêm dos pequenos atos que podem ser durante a troca de bolsa ou limpeza da pele. Da mesma forma, estudos ${ }^{(15,16)}$ referem que o uso de equipamento coletor $\mathrm{e}$ a incapacidade de controle de gases e fezes alteraram a imagem que o indivíduo tem de si, passando a experimentar sentimentos negativos sobre o seu corpo e sua inferioridade, alterando sua autoestima e provocando alterações emocionais.

Ainda no que tange ao uso do equipamento, autores ${ }^{(17)}$ relatam que a limpeza da bolsa coletora é realizada várias vezes ao dia pelos pacientes, geralmente após cada evacuação. Assim, a troca é, também, um cuidado importante, principalmente para a fixação da bolsa coletora na pele, e este cuidado faz com que os pacientes se sintam mais seguros e consigam desempenhar suas atividades diárias.

$\mathrm{O}$ medo e a insegurança, bem como sentimentos ligados à vergonha e ao sofrimento, foram identificados em estu$\mathrm{do}^{(18,19)}$. Esses sentimentos puderam ser identificados como determinantes na nova condição emocional, refletindo diretamente na sua vida social, amorosa e laboral desses indivíduos.

Seguindo o raciocínio, autores ${ }^{(3)}$ relatam que os pacientes, ao serem informados da necessidade da confecção de uma estomia, apresentam sentimentos, como: ansiedade, incertezas, medo do diagnóstico, raiva, desespero, angústia, rejeição 
e impotência. Após a confecção da estomia, foram identificados problemas com autoestima diminuída devido à alteração da imagem corporal, em que os pacientes precisam se adaptar a um novo corpo e a uma nova forma de eliminação das fezes. Outros sentimentos, como depressão, angústia, insegurança, revolta, desgosto, ódio, raiva, repulsa, agressividade, não aceitação, autor rejeição, luto, perda do autocontrole e da identidade também foram observados.

No que se refere à adaptação, a necessidade de adequação da alimentaçãoo ${ }^{(17)}$ e o ajuste em relação à perda do controle sobre suas eliminações e flatos ${ }^{(19)}$ puderam ser identificados como as principais dificuldades enfrentadas. Ainda, existem alterações relacionadas ao vestuário, perda de confiança, independência e dignidade, além de dificuldade para viajar devido à mudança de hábitos e alteraçóes de privacidade.

A sexualidade também sofreu adaptação devido à confecção da estomia ${ }^{(16,18,19)}$. Os pacientes relataram alterações devido à diminuição da autoestima, autoimagem prejudicada e vergonha ou medo da não aceitação pelo parceiro em função da presença do estoma como parte do corpo. Frente a isso, a sexualidade de pessoas estomizadas é afetada a partir da perda da libido e impotência, pelo medo de a bolsa extravasar liberando fezes e odor durante o ato sexual. Essas questões com a sexualidade podem ser causadas por problemas psicológicos ou fisiológicos, com mudanças nos relacionamentos conjugais ${ }^{(3)}$.

Ressalta-se que grande parte dos pacientes estomizados negou praticar atividade sexual. A técnica cirúrgica de Miles é a mais comum, levando, diversas vezes, os pacientes à disfunção erétil, ocorrendo, no momento da cirurgia, a ressecção de vasos sanguíneos e terminações nervosas responsáveis por esta função nos homens. Já nas mulheres, a remoção do tumor de cólon e reto leva ao encurtamento do canal vaginal, por este ser muito próximo do reto, acarretando a perda da libido e dispareunia. Esses fatores podem afastar, assim, as possibilidades de vida sexual, levando os pacientes a sentimentos de isolamento, vergonha e
De acordo com a política de saúde do trabalhador, a pessoa

com estoma tem

$$
\text { direito a retornar }
$$

as suas atividades

laborais em

adequada condição

para atender suas

limitações físicas.

desinteresse sexual, sendo necessário orientação por um profissional de saúde ${ }^{(20)}$.

Cabe lembrar que, autores ${ }^{(21)}$ afirmam que, devido ao desconforto com a aparência, o paciente estomizado se sente envergonhado frente ao outro. Sendo assim, apresenta baixa autoestima, o que leva ao isolamento, sendo esta uma forma de ocultar o estoma, isto é, não se relacionando com o parceiro.

No mesmo sentido, autores ${ }^{(12)}$ fazem referência à falta de apoio psicológico sobre as questões sexuais à clientela estomizada como uma limitação para a retomada da vida sexual. Assim, a sexualidade do paciente estomizado é afetada pela autoestima, tendo a imagem corporal abalada e apresentando medo e ansiedade.

Em relação às atividades laborais, estudos $^{(3,20)}$ destacaram que os pacientes que possuíam vínculo empregatício preferiram se afastar em definitivo das atividades laborais, mesmo após a sua re- cuperação, preferindo receber o benefício previdenciário. Autores ${ }^{(20)}$ relatam sobre a dificuldade de inserção desse indivíduo no mercado de trabalho, quando se encontram desempregados.

De acordo com a política de saúde do trabalhador, a pessoa com estoma tem direito a retornar as suas atividades laborais em adequada condição para atender suas limitações físicas. Sendo assim, essa pessoa está respaldada legalmente pela Política Nacional para a Integração da Pessoa Portadora de Deficiência, podendo utilizar a reserva de vagas de emprego ${ }^{(15)}$.

O efeito da estomia na vida laboral e a redução da capacidade para o trabalho fazem parte dos limites e consequências decorrentes das alteraçôes fisiológicas e anatômicas. Os cuidados com as bolsas coletoras fazem o paciente estomizado perceber suas limitações, alterando suas atividades do dia a dia, relacionadas com medo e constrangimento no ambiente de trabalho.

Esses sentimentos e adequaçóes ao estilo de vida, muitas vezes, puderam ser associados ao isolamento social das pessoas estomizadas $^{(2,3,17)}$.

Com relação ao isolamento social, autores ${ }^{(13)}$ apontam que os pacientes passaram por longos períodos de privação de momentos de atividades de lazer com a família e amigos, em que os estomizados permaneciam no domicílio, principalmente, por medo de a bolsa coletora soltar e extravasar conteúdo eliminado.

Autores $^{(2)}$ referem que o isolamento social, também conhecido como "morte social", advém do medo de serem estigmatizados e excluídos da sociedade, diante da alteração na sua imagem corporal e o não controle das fezes e flatos. Os autores também revelam que muitos sentem dificuldades de voltar às atividades laborais devido à insegurança, autoimagem desconfigurada, problemas de adaptação e aos cuidados com a bolsa coletora.

Os estudos demonstram o processo de transformação que a estomização provoca na vida e no psicológico dos pacientes. Os sentimentos negativos vivenciados têm influência direta e podem ser identificados em diversas áreas da vida, tanto no campo 
das relações interpessoais quanto em suas atividades laborais.

Autores $^{(15)}$ destacam que a estomização apresenta, em quem a vive, sentimentos positivos, negativos e de aceitação, representados pela possibilidade de continuação da vida, pelas dificuldades pelas perdas do controle esfincteriano e pela adaptação frente à sua condição de saúde.

\section{Medidas de enfrentamento no pro- cesso de reabilitação da pessoa com estomia}

Nesta categoria, foram relatadas dificuldades de enfrentamento por sentimentos de desprezo, nojo e vergonha da exposição de fezes para outras pessoas, principalmente no momento de esvaziar ou fazer a limpeza da bolsa coletora e do odor desagradável. Ainda, nota-se muita preocupação de a bolsa coletora soltar e vazar conteúdo fecal.

Após a confecção da colostomia, os indivíduos vivenciam a experiência de se ver como um ser diferente, o que lhes impõe um ajuste de sua imagem e autoconceito. Além disso, observa-se que os impactos das mudanças relacionadas à estomia perduram por, pelo menos, cinco anos após a cirurgia, com destaque para o comprometimento físico, social e financeiro nos primeiros seis meses. Ressalta-se, ainda, que a variável tempo de estomizado se apresenta diretamente relacionada à qualidade de vida ${ }^{(18,22)}$.

Esses e outros ajustes, bem como o enfrentamento de várias complicações, são temas que precisam ser abordadas pelo profissional de enfermagem para que o paciente consiga superar seus próprios estigmas e realizar o autocuidado. Sendo assim, o profissional de enfermagem é uma figura de apoio à transição para novas experiências vividas pelos pacientes ${ }^{(18)}$.

Frente a isso, autores ${ }^{(14)}$ discorrem que o paciente possui sua individualidade, realizando o enfrentamento de forma singular. Isto quer dizer que, cada um passa por seu momento e sua forma de enfrentamento e adaptação à sua nova condição, vivendo com o sofrimento, a dor, a incerteza, mitos e medos, sendo a equipe de enfermagem indispensável no acompanhamento ao estomizado.

Pesquisas demonstraram que os profissionais de enfermagem perceberam que a pessoa com estomia intestinal, assim como a família, inicialmente, apresenta sentimentos de rejeição, medo, distúrbio de autoimagem, baixa autoestima, e que esses sentimentos negativos dificultam o aprendizado do autocuidado e da adaptação à nova condição ${ }^{(4)}$.

Estudo $^{(4)}$ revela que os pacientes orientados no pré-operatório evoluíram melhor diante da situação. A consulta de enfermagem para a realização da demarcação no abdômen do estoma reduz problemas de fixação do dispositivo coletor, e revela ao paciente como será confeccionado o estoma e qual o seguimento do intestino será exteriorizado. Outras informações, como o tempo de permanência, o tipo de efluente que será eliminado na bolsa, os flatos, possíveis complicações na pele, impactos na imagem corporal, vestuário, alimentação e sexualidade já preparam o paciente para a reabilitação e a realização do autocuidado.

Relacionando pontos positivos, a aceitação da estomia se constitui um produto de estratégia eficaz de enfrentamento para tratamento da doença de base. Embora vários fatores dificultem o processo adaptativo do estomizado, o tratamento de comorbidades é visto de forma positiva e esperançosa, sendo o entendimento da qualidade de vida um fator subjetivo e individual ${ }^{(23)}$.

Cabe ressaltar que, segundo autores ${ }^{(19)}$, o progresso para a completa reabilitação acontece quando a pessoa estomizada consegue aceitar as mudanças relacionadas ao uso do equipamento coletor e a perda da continência fecal. Sendo assim, a implementação de tecnologias oclusoras e de irrigação para pessoas com colostomias, por exemplo, pode oferecer ampla escolha de gestão do autocuidado, melhorando a imagem corporal, recuperando a continência por até 12 horas e reduzindo o estresse desencadeado por essa situação.

A irrigação tem por finalidade esvaziar o intestino de gases, muco e fezes, ajudando o estomizado a regularizar o trânsito intestinal, dando a ele a possibilidade de permanecer sem a bolsa coletora por algumas horas, contribuindo para a melhoria de seu bem-estar e sua qualidade de vida ${ }^{(23)}$.

Outra medida de enfrentamento foi a religião. Em estudo ${ }^{(16)}$ observou-se que a religião, a fé e a crença são consideradas importantes ferramentas para o processo de sobrevivência e enfrentamento, pois, através da religião, pacientes estomizados encontram forças e apoio social para o enfrentamento da dor e de suas angústias na vida diária, produzindo alívio do sofrimento relacionado tanto à sua nova condição de estomizado, quanto ao tratamento da doença que o levou a ficar estomizado.

Ainda sobre a fé e a religiosidade, autores ${ }^{(1)}$ discorrem que o bem-estar espiritual pode ser abalado após a estomização. Essa fala se evidenciou quando foi relatada a fé e a religiosidade como fatores relacionados à pessoa. Para o paciente que tem fé, o processo de transição é facilitado, permitindo que suas forças se voltem para a saúde e a autonomia, melhorando a reflexão acerca do viver e facilitando a ressignificação da vida.

Para o enfrentamento, são utilizadas por pacientes estomizados as redes de apoio. O conhecimento a respeito dessas redes sociais de apoio no cuidado pode auxiliar na melhoria da qualidade de vida, permitindo que os pacientes estomizados consigam enfrentar melhor sua nova situação. Por este motivo, é importante que o enfermeiro conheça e valorize essas redes a fim de observar a pessoa com estomia e seu contexto social.

Durante todo o processo de reabilitação, o estomizado busca uma rede de apoio - amigos, familiares, profissionais de saúde e outros pacientes estomizados - que possa ajudar para o enfrentamento dos desafios relacionados à presença do estoma. Profissionais de enfermagem são essenciais no aprendizado do autocuidado, tendo como resultados a segurança e a convivência de forma harmônica com a nova condição ${ }^{(24)}$.

Segundo estudo ${ }^{(25)}$, os pacientes estomizados acreditam ser indispensável o apoio dos familiares, especialmente após a cirurgia de estomização, pois é nesse período em que estão vivendo momentos de intensa desordem emocional, sendo necessá- 
rio um novo aprendizado para cuidar de si.

Corroborando ao contexto, autores ${ }^{(26)}$ apontam o apoio de associações e grupos de pessoas com estomias como um lugar de troca e aprendizagem, onde há transferência de experiências entre profissionais e os próprios estomizados. Acredita-se que esse espaço de convivência possa favorecer a aceitação da pessoa à sua nova condição de estomizado.

A pessoa estomizada, além de ter o seu equilíbrio afetado por uma doença de base, sofre as repercussões emocionais de ser submetida à cirurgia de estomização, a partir da qual ela terá de lidar com o novo. Esses pacientes precisam de redes fortes de apoio para a adaptação e recuperação biopsicossocial, em que tanto os profissionais de saúde, os familiares, amigos e até mesmo outros pacientes fazem parte do processo de enfrentamento e adaptação.

Ainda sobre o enfrentamento e a reabilitação, autores ${ }^{(27)}$ apontam que é um processo longo para ser alcançado, sendo contemplado em diversas dimensões. Este não se limita apenas em recuperar funções perdidas ou alteradas, mas abrange aspectos físicos, psicológicos, sociais, espirituais, econômicos e políticos, sendo a reabilitação e a adaptação os focos dos pacientes após sua nova condição de vida.

\section{0 cuidado de enfermagem: apoio pro-} fissional relacionado ao autocuidado

Nesta categoria, buscou-se, na literatura, ações de apoio ao autocuidado e a reabilitação desenvolvidas por enfermeiros. Embora a equipe de enfermagem esteja diretamente ligada a ações de educação em saúde, aconselhamento, orientações e cuidados, poucos estudos relacionam os sentimentos negativos vividos por pacientes estomizados às dificuldades de adaptação.

Nesse contexto, estudo ${ }^{(17)}$ afirma que os profissionais de enfermagem têm relevante papel no processo de reabilitação, atuando junto à pessoa estomizada e à família de maneira integral, individualizada e sistematizada, com a finalidade de melhorar a qualidade de vida. Para tanto, é necessário que enfermeiros ofereçam assistência, atendendo as necessidades biológicas e
A pessoa estomizada,

além de ter o seu

equilíbrio afetado por

uma doença de base,

sofre as repercussóes

emocionais de ser

submetida à cirurgia

de estomização, a

partir da qual ela

terá de lidar com o

novo. Esses pacientes

precisam de redes

fortes de apoio

para a adaptação

e recuperação

biopsicossocial,

em que tanto os

profissionais de saúde,

os familiares, amigos

e até mesmo outros

pacientes fazem

parte do processo

de enfrentamento $\mathrm{e}$

adaptação. psicossociais e favorecendo ao indivíduo um retorno às atividades rotineiras o mais brevemente possível.

Autores ${ }^{(13)}$ destacam que a assistência de enfermagem à pessoa estomizada deve começar já no pré-operatório, através de entrevista, exame físico, diagnóstico e planejamento das ações de enfermagem. É necessário que a equipe prepare o paciente física e emocionalmente, avaliando sua capacidade emocional e física para a realização do autocuidado.

A enfermagem é a categoria profissional da área de saúde que destina mais tempo com cuidados aos pacientes estomizados. Estes cuidados são reforçados desde a orientação da utilização do dispositivo coletor, do manejo da pele, higienização, irrigação intestinal, apoio às questões de adaptação para o retorno à vida social, laboral e atividade sexual. O enfermeiro é o protagonista nos processos de reabilitação dos pacientes pós-estomia. Autores ${ }^{(12)}$ apontam que, após o procedimento cirúrgico, o paciente terá de assumir a tarefa de cuidados específicos com sua nova condição de estomizado, cuidados estes que, antes, não eram necessários.

A equipe de enfermagem se destaca na assistência às pessoas com estomia desde o pré-operatório até a chegada dos pacientes aos serviços especializados e cuidados domiciliares. Para isso, é preciso que os enfermeiros formulem sua assistência pautada no reconhecimento das subjetividades e na interpretação de que o processo saúde-doença é um resultado psicossocial e cultural que se altera conforme as interações sociais ${ }^{(2)}$.

Relacionando o cuidado de enfermagem à vida sexual do estomizado, autores $^{(21)}$ discorrem que esta influencia diretamente na qualidade de vida e, por este motivo, é necessário que o enfermeiro fale a respeito com o paciente estomizado, a fim de oferecer assistência integral e personalizada. Para isto, é necessário que o enfermeiro desenvolva escuta ativa e atendimento individualizado, levando em conta os sentimentos relativos ao processo vivido por cada um.

Artigos abordam a necessidade de atuação da equipe de enfermagem para melho- 
rar o autocuidado de estomizados, destacando a educação em saúde e as práticas que podem facilitar a reabilitação de pacientes, como cuidados com a bolsa coletora, manejo com a pele, restrições alimentares para evitar o aumento de gazes e a mudança na consistência do conteúdo fecal ${ }^{(24) .}$

Segundo estudo ${ }^{(3)}$, o enfermeiro, em conjunto com a equipe multidisciplinar, deve planejar desde a alta hospitalar do paciente até seu acompanhamento no polo de atenção à saúde da pessoa estomizada. Devem ser oferecidas intervenções educativas sobre o autocuidado, equipamentos coletores e adjuvantes, cuidados com a pele periestoma, assistência especializada multiprofissional para enfrentamento e reabilitação. Os autores afirmam, ainda, que a enfermagem pode contribuir na assistência especializada ao estomizado e à sua família no gerenciamento das situações ou alterações, fornecendo suporte profissional às necessidades individuais de cada paciente.

Os artigos analisados não incluem, de forma específica em seus planos de cuidados e orientações, o acompanhamento psicológico e apoio emocional para a melhor adaptação de vida dessa clientela. $\mathrm{O}$ autocuidado é relacionado apenas à prática efetiva em troca de dispositivos, avaliação do estoma e da pele periestoma. Nesses estudos, a reabilitação está relacionada apenas ao bom manejo do estoma e ao retorno às atividades diárias, deixando para segundo plano as dificuldades de aceitação e adap- tação resultantes dos sentimentos vividos por esses pacientes durante este processo.

Para autores ${ }^{(28)}$, a consulta de enfermagem é essencial para a readaptação de pacientes estomizados, pois, além de oferecer suporte necessário para o tratamento, oferece um sentido, guiando o paciente para a aceitação da estomia, para a compreensão das alterações ocorridas em seu corpo, salientando que os estomizados que frequentaram as consultas de enfermagem são exatamente aqueles que tiveram a melhor adaptação à sua nova condição. Assim, percebe-se que o enfermeiro é fundamental na recuperação terapêutica.

Autores $^{(15)}$ descrevem que o enfermeiro tem papel importante no processo de orientação do estomizado, pois possui competência e ferramentas assistenciais, como a Sistematização da Assistência de Enfermagem, para identificar as dificuldades de adaptação destes clientes à sua condição de estomizado, além de propor, em conjunto com o paciente e a equipe, ações que objetivam a superação de dificuldades.

A implantação das etapas do processo de enfermagem amplia os conhecimentos e os cuidados dirigidos para os estomizados, identificando os diagnósticos de enfermagem para esses indivíduos. A partir daí, é possível formular planejamento e implementação de cuidados que podem permitir a esses pacientes desenvolverem resiliência e adaptação ao seu novo contexto de vida ${ }^{(28)}$.
Ainda no que tange ao apoio profissional, autores ${ }^{(2)}$ discorrem que a enfermagem estabelece relações que oferecem forte apoio, evidenciando um trabalho desenvolvido em prol do bem-estar desses pacientes. A enfermagem pode tecer teias de relações interpessoais com estomizados e familiares, favorecendo a troca de experiência para melhorar o cuidado.

\section{CONCLUSÃO}

Os sentimentos vivenciados após a confecção da estomia influenciaram na adaptação desses pacientes. As mudanças da dinâmica corporal e nas atividades de vida diária impostas pela confecção do estoma acarretaram no sujeito momentos de depressão, medo, tristeza, isolamento social e afastamento da atividade laboral.

A pessoa com estomia, além de ter seu equilíbrio afetado por uma doença de base, sofre as repercussões de ser submetida à cirurgia para confecção de um estoma, necessitando de ações de apoio para reabilitação, tendo a enfermagem relevante papel nesse processo. O enfermeiro, em conjunto com a equipe multidisciplinar, precisa desenvolver além de planos de cuidados e orientações, acompanhamento psicológico e apoio emocional para que a pessoa com estomia tenha uma melhor adaptação, reabilitação e retorno para as atividades de vida diária.

\section{REFERÊNCIAS}

1. Mota MS, Gomes GC, Petuco VM. Repercussões no processo de viver da pessoa com estoma. Texto Contexto Enferm [Internet]. 2016 [acesso em 28 jul 2017]; 25(1):1-13. Disponível em: http://www.scielo.br/pdf/tce/v25n1/pt_0104-0707tce-25-01-1260014.pdf.

2. Simon BS, et al. "Sempre ajudando em uma coisa ou outra": rede social da família da pessoa com estomia. Rev Eletr Enferm. 2015;17(2):370-378. http://dx.doi.org/10.5216/ree. v17i2.29786.

3. Teles AAS, et al. Mudanças Físicas, psicossociais e os sentimentos gerados pela estomia intestinal para o paciente: Revisão Integrativa. Rev Enferm UFPE on line [Internet]. 2018 fev [acesso em 28 jul 2018]; 11(2):1062-1072. Disponível em: https:// pdfs.semanticscholar.org/3732/5ddd52d45f75ef9aaefe2a08ff41a5640390.pdf.

4. Ardigo FS, Amante LN. Conhecimento do profissional acerca do cuidado de enfermagem à pessoa com estomia intestinal e família. Rev Texto Contexto Enferm [Internet]. 2013 [acesso em 02 jun 2018]; 22(4):1064-1071. Disponivel em: http://www.scielo.br/pdf/tce/v22n4/24.pdf.

5. Floriani FM, Marcante MDS, Braggio L. A. Auto estima e auto imagem: A relação com a estética. Univali [Internet]. 2010 [acesso em 08 set 2018]; 1-15. Disponível em: http://siaibib01.univali. br/pdf/Flavia\%20Monique\%20Floriani,\%20M\%C3\%A1rgara\%20 Dayana\%20da\%20Silva\%20Marcante.pdf.

6. Broome M. Integrative literature reviews for the development 


\section{artigo}

Citino, H.P.; Andrade, P.C.S.T.; Kestenberg, C.C.F.; Caldas, C.P.; Santos, C.N.; Ribeiro, W.A.

Repercussões emocionais e processos adaptativos vividos por pessoas estomizadas

\section{REFERÊNCIAS}

of concepts. In: Rodgerd BL, Knalf KA, editors. Concept development in nursing: foundations, techniques and applications. Philadelphia (USA): W. B Saunders Company, P.; 2000. P. 231-50

7. Botelho LLR, Cunha CCA, Macedo M. O método da revisão integrativa nos estudos organizacionais. Rev Gestão e Sociedade [Internet]. 2011 [acesso em 02 jun 2018]; 5(11):121-136. Disponivel em: https://www.gestaoesociedade.org/gestaoesociedade/article/view/1220/906.

8. Santos VLCG. Aspectos epidemiológicos dos estomas. Revista Estima [Internet]. 2007 [acesso em 10 jul 2018]; 5(1):115-125. Disponível em: http://revistaestima.com.br/index.php/estima/ article/view/207.

9. Mendes KDS, Silveira RCC, Galvão CM. Revisão integrativa: métodos de pesquisa para a incorporação de evidências na saúde e na enfermagem. Texto Contexto enferm [Internet]. 2008 [acesso em 25 jul 2018]; 17(4):758-764. Disponível em: $\quad$ http://www.scielo.br/scielo.php?script=sci_arttext\&pid=s0104-07072008000400018.

10. Souza MT, Silva MD, Carvalho R. Revisão integrativa: o que é e como fazer. Rev Einstein [Internet]. 2010 [acesso em 25 jul 2018]; 8(1):102-106. Disponivel em: http://www.scielo.br/pdf/ eins/v8n1/pt_1679-4508-eins-8-1-0102.pdf.

11. Polit DF, Beck CT, Hungler BP. Fundamentos de pesquisa em enfermagem: Métodos, avaliação e utilização. 5.ed. Porto Alegre: Artmed; 2004. p. 309-356.

12. Coelho AR, Santos SF, Poggetto MTD. A estomia mudando a vida: enfrentar para viver. Rev Min Enferm [Internet]. $2013 \mathrm{abr/}$ jun [acesso em 10 jun 2018]; 17(2):258-267. Disponivel em: http://www.reme.org.br/artigo/detalhes/649.

13. Ramos RCA, et al. Pacientes com derivações urinárias: uma abordagem sobre as necessidades humanas básicas afetadas. Rev enferm UERJ [Internet]. 2013 jul/set [acesso em 10 nov 2018]; 21(3):337-42. Disponivel em: www.epublicacoes.uerj. br/ index.php/enfermagemuerj/article/view/7516/5439.

14. Wild CF, et. al., Educação em saúde com estomizados e seus familiares: Possibilidade para melhor qualidade de vida. Rev. Enf. UFSM [Internet]. 2016 [acesso em 10 jun 2018]; 6(2). Disponível em: https://periodicos.ufsm.br/reufsm/article/view/20071.

15. Maurício VC, Souza NVDO, Lisboa MT. Determinantes biopsicossociais do processo de inclusão laboral da pessoa estomizada. Rev Bras Enferm [Internet]. 2014 [acesso em 21 jul 2018]; 67(3):415-421. Disponivel em: http://www.scielo.br/pdf/reben/ v67n3/0034-7167-reben-67-03-0415.pdf.

16. Aguiar JC, et al. Aspectos sociodemográficos e clínicos de estomizados intestinais provisórios. REME [Internet]. 2017 [acesso em 18 ago 2019]; 21:1006-1013. Disponivel em: http://www. reme.org.br/artigo/detalhes/1149.

17. Barba PD, et al. Demandas de cuidados de pacientes oncológicos estomizados assistidos na atenção primária à saúde. Rev. Enferm. UFPE [Internet]. 2017 [acesso em 18 ago 2019]; 11(8):3122-3129. Disponivel em: https://pdfs.semanticscholar.org/7beb/cac053d0d7212a6d403bd53c7b5d- d5cb969d.pdf?_ga=2.105892227.1042138975.1579487404666848754.1579487404.

18. Freire DA, et al. Autoimagem e autocuidado na vivência de pacientes estomizados: o olhar da enfermagem. REME [Internet]. 2017 [acesso em 18 ago 2019]; 21:1019. Disponível em: http://www.reme.org.br/artigo/detalhes/1155.

19. Silva CRDT, et al. Qualidade de vida de pessoas com estomias intestinais de eliminação. Acta Paul Enfer [Internet]. 2017 [acesso em 13 jun 2018]; 30(2):144-151. Disponivel em: http://www. scielo.br/pdf/ape/v30n2/1982-0194-ape-30-02-0144.pdf.

20. Vera SO, et al. Sexualidade de pacientes com estomias intestinais de eliminação. Rev. Fund Care Online [Internet]. 2017 [acesso em 29 jul 2018]; 9(2):495-502. Disponivel em: http:// www.seer.unirio.br/index.php/cuidadofundamental/article/ view/5451/pdf_1.

21. Goulart MB, et al. A sexualidade do paciente estomizado no discurso do Enfermeiro. REME [Internet]. 2017 [acesso em 10 jun 2018]; 21:e1041. Disponivel em: https://www.reme.org.br/ artigo/detalhes/1179.

22. Torres CRD, et al. Qualidade de vida de pessoas estomizadas: revisão integrativa. Rev enferm UFPI [Internet]. 2015 [acesso em 28 jul 2018]; 4(1):117-122. Disponível em: https://pesquisa. bvsalud.org/portal/resource/pt/bde-30843.

23. Costa IKF, et al. Distúrbio na imagem corporal: diagnóstico de enfermagem e características definidoras em pessoas ostomizadas. AQUICHAN [Internet]. 2017 [acesso em 10 jun 2018];17(3):270-283. Disponivel em: https://www.researchgate.net/publication/319500228_Disturbio_na_imagem_ corporal_diagnostico_de_enfermagem_e_caracteristicas_ definidoras_em_pessoas_ostomizadas.

24. Albuquerque AFLL, et al. Tecnologia para o autocuidado da saúde sexual e reprodutiva de mulheres estomizadas. Rev. Bras. Enferm. 2016;69(6):1099-1106. http://dx.doi. org/10.1590/0034-7167-2016-0302.

25. Souza JL, et al. O prepare do familiar para o cuidado à pessoa com estomia. Rev Enfer UFPE [Internet]. 2013 [acesso em 28 jul 2018]; 7(1):649-656. Disponivel em: http://periodicos.ufpe.br/ revistas/revistaenfermagem/article/download/10276/10915.

26. Rodrigues AS, Budó MLD, Simon BS, et al. As redes sociais de apoio no cuidado às pessoas com estomias: Revisão Bibliográfica. Saúde (Santa Maria),2013(39)(1):33-42.

27. Marques GS, et al. A vivência de pessoas com estomia intestinal no grupo de apoio em um Hospital Universitário. Revista HUPE [Internet]. 2016 [acesso em 10 jun 2018]; 15(2). Disponivel em: https://www.e-publicacoes.uerj.br/index.php/ revistahupe/article/view/28235.

28. Melo MDM, et al. Revisão integrativa das características definidoras do diagnóstico de enfermagem: disposição para resiliência melhorada em ostomizados. Rev. Min. Enferm [Internet]. 2015 [acesso em 21 jul 2018]; 19(3):779-785. Disponivel em: https://pesquisa.bvsalud.org/portal/resource/pt/ bde-28193. 